\title{
Rechenschwierigkeiten und Rechenstörungen
}

\author{
Multimodales Therapieprogramm für Kinder \\ und Jugendliche
}

Hartmut E. Bernart \& Jakob Weinig (2020). Beltz, Weinheim. 334 Seiten, ISBN 978-3-621-28698-5, $€ 39,95$

„Ein leitliniengerechtes und wissenschaftlich fundiertes Instrument [...], das zugleich aus der Praxis stammt“. Das Vorwort zu „Rechenschwierigkeiten und Rechenstörungen" verspricht Großes. Hartmut E. Bernart und Jakob Weinig sind Integrative Lerntherapeuten nach FiL, die über langjährige Praxiserfahrung verfügen und nun ihr Lerntherapiekonzept für Rechenstörungen in einem Buch zusammengestellt haben. Dieses enthält einen DownloadLink für eine E-Book-Variante mit ca. 380 Seiten Zusatzmaterial. Doch hält das Buch, was es verspricht?

\section{Aufbau und Inhalt des Buches}

Das Buch ist in drei Teile gegliedert: Das Störungsbild, die funktionelle Übungsbehandlung und die Psychologische Arbeit. Im ersten Teil zum Störungsbild werden Definition, Symptomatik, Ätiologie und Diagnostik von Rechenschwierigkeiten und Rechenstörungen beschrieben. Die Autoren beziehen sich in diesem theoretischen Teil auf wissenschaftliche Studien, bestehende Modelle der Zahlenverarbeitung und mathematischen Entwicklung, sowie auf ein modifiziertes Lern-Wirkungsgefüge nach Betz \& Breuninger (1998). Aus diesen theoretischen Grundlagen zusammengesetzt wird ein "Haus des Rechenerwerbs“ präsentiert, an dem sich der Therapieansatz orientiert.

Im zweiten Teil zur funktionellen Übungsbehandlung wird das Therapiekonzept dargestellt. Der Begriff der ,funktionellen Übungsbehandlung “ ersetzt hierbei durchgehend den Begriff der Lerntherapie und versteht sich als Alleinstellungsmerkmal. Dieser Teil des Buches umfasst die Grundlagen der funktionellen Übungsbehandlung sowie eine detaillierte Durchführungsanleitung und schließlich die Vorstellung von 11 Therapiestufen, die von der Addi- tion im Zahlenraum 10 bis $\mathrm{zu}$ Ergänzungsaufgaben der Multiplikation und Division bis 100 reichen (Materialen für weiterführende Therapieinhalte finden sich jedoch im E-Book).

Im dritten Teil zur Psychologischen Arbeit werden zunächst allgemeine Grundlagen der psychologischen Arbeit in der Lerntherapie beschrieben. Darauf folgen konkrete Anweisungen und Handlungsempfehlungen für die Arbeit mit Kindern/Jugendlichen, Eltern und Lehrkräften. Neben der Psychoedukation wird hier jeweils auch auf die Arbeit an dysfunktionalen Kognitionen, Leistungserwartungen und Selbstkonzept eingegangen.

\section{Einschätzung der Stärken und Schwächen des Buches}

Die Autoren Bernart und Weinig haben sich mit diesem Buch viel vorgenommen. Dafür haben sie umfassend recherchiert und Evidenz aus der Forschung und aus relevanten Metaanalysen in ihre Ausführungen miteinbezogen. Zudem wird Bezug zur „S3-Leitlinie zur Diagnostik und Behandlung der Rechenstörung" (AWMF, 2018) genommen. In dieser Leitlinie ist auf Basis von wissenschaftlicher Evidenz und Expertenkonsens festgehalten, wie eine Rechenstörung festzustellen und zu behandeln ist.

Im ersten Teil des Buches zum Störungsbild wird auf den wissenschaftlichen Hintergrund am meisten Bezug genommen. Darunter leidet jedoch die Verständlichkeit. Dieser erste Teil enthält viele Fachbegriffe aus der wissenschaftlichen Literatur, die nicht immer verständlich erklärt werden. Durch die Fachsprache und die fehlenden Übergänge zwischen den Abschnitten ist es teils schwierig, dem roten Faden in diesem Teil zu folgen.

Anregender zu lesen ist hingegen die Beschreibung der funktionellen Übungsbehandlung im zweiten Teil. Hier wird ein Therapiekonzept aus der Praxis mit Evidenz aus Literatur und Leitlinien unterfüttert, und somit nicht nur 
detailliert beschrieben, sondern auch gut begründet. Mit dem Material aus dem E-Book stehen zudem Grafiken und Arbeitsmaterial für die praktische Umsetzung zur Verfügung. Wünschenswert wäre ein noch stärkerer Einbezug der mathematischen Basiskompetenzen (z.B. Mengenerfassung, Zahlwortreihe), die bei vielen Kindern mit Rechenstörungen die größte Schwierigkeit darstellen und vor der Erarbeitung der Addition im Zahlenraum 10 gefestigt sein sollten. Positiv fällt auf, dass die Autoren die Diagnostik als notwendigen Teil der lerntherapeutischen Arbeit verstehen und diese einen selbstverständlichen Eingang in das Konzept findet. Vorgesehen sind Vor- und Nachtests für jeden der beschriebenen Therapieschritte.

Eine willkommene Ergänzung des Therapiekonzepts findet sich im letzten Teil zur Psychologischen Arbeit. Hier zeigt sich deutlich, dass integrative Lerntherapeuten die Feder führen, die neben der hauptsächlichen Arbeit am Symptom auch das psychische Wohlbefinden des Kindes und dessen Interaktionen mit seinem Umfeld im Blick haben. In diesem Teil werden konkrete Methoden für Lerntherapeut_innen aufgezeigt, wie sowohl mit Kindern als auch Eltern und Lehrkräften gearbeitet werden kann, um das seelische Wohlbefinden des Kindes im Rahmen der Lerntherapie zu verbessern.

Die Autoren betonen, dass ihr Therapieprogramm evidenzbasiert ist und auf wissenschaftlichen Erkenntnissen sowie der S3-Leitlinie beruht. Letztlich ist das Programm zwar in einen wissenschaftlichen Kontext eingebettet, jedoch nicht wissenschaftlich evaluiert (d.h. auf seine Wirksamkeit überprüft). Dies wäre laut der S3-Leitlinie eine Voraussetzung für die Empfehlung des Programms und ist der Tatsache zu schulden, dass es eben aus der Praxis stammt. Dies ist keine Kritik an den Programminhalten, sollte aber berücksichtigt werden, wenn eine Passung zur S3-Leitlinie angestrebt wird.

\section{Fazit}

„Rechenschwierigkeiten und Rechenstörungen“ ist ein Buch aus der Lerntherapie für die Lerntherapie. Bernart und Weinig stellen ein in der Praxis erprobtes Therapieprogramm vor, welches detailgenau beschrieben und vermittelt wird. Die Einbettung des Programms in einen wissenschaftlichen Rahmen ist $\mathrm{zu}$ begrüßen, macht die Lektüre jedoch holpriger als dies von einem rein praktischen Therapiemanual zu erwarten wäre. Dies könnte für Leser_innen eine Herausforderung darstellen, die mithilfe des Buches einen Einblick in das Störungsbild und die wissenschaftlichen Grundlagen erhalten möchten. Wer jedoch eine klar strukturierte Handlungsanweisung für die Behandlung von Rechenstörungen und die psychologische Arbeit in der Lerntherapie sucht, wird hier durchaus fündig. Somit ist das Buch insbesondere für angehende Lerntherapeut_innen zu empfehlen, die in die Therapie von Rechenstörungen einsteigen möchten.

\section{Literatur}

Betz, D. \& Breuninger, H. (1998). Teufelskreis Lernstörungen. Theoretische Grundlegung und Standardprogramm (5. Aufl.). Weinheim: Beltz.

Arbeitsgemeinschaft der Wissenschaftlichen Medizinischen Fachgesellschaften e.V. (2018). S3-Leitlinie: Diagnostik und Behandlung der Rechenstörung. Verfügbar unter https://www.awmf.org/ uploads/tx_szleitlinien/028-046l_S3_Rechenst\%C3\%B6rung2018-03_1.pdf [01.06.2020]. 